\title{
Lyapunov exponents for small aspect ratio Rayleigh-Bénard convection
}

\author{
J. D. Scheel* and M. C. Cross \\ Department of Physics, California Institute of Technology 114-36, Pasadena, California 91125, USA
}

(Received 1 March 2006; revised manuscript received 12 October 2006; published 4 December 2006)

\begin{abstract}
Leading order Lyapunov exponents and their corresponding eigenvectors have been computed numerically for small aspect ratio, three-dimensional Rayleigh-Benard convection cells with no-slip boundary conditions. The parameters are the same as those used by Ahlers and Behringer [Phys. Rev. Lett. 40, 712 (1978)] and Gollub and Benson [J. Fluid Mech. 100, 449 (1980)] in their work on a periodic time dependence in RayleighBenard convection cells. Our work confirms that the dynamics in these cells truly are chaotic as defined by a positive Lyapunov exponent. The time evolution of the leading order Lyapunov eigenvector in the chaotic regime will also be discussed. In addition we study the contributions to the leading order Lyapunov exponent for both time periodic and aperiodic states and find that while repeated dynamical events such as dislocation creation/annihilation and roll compression do contribute to the short time Lyapunov exponent dynamics, they do not contribute to the long time Lyapunov exponent. We find instead that nonrepeated events provide the most significant contribution to the long time leading order Lyapunov exponent.
\end{abstract}

DOI: 10.1103/PhysRevE.74.066301

PACS number(s): 47.54. $-\mathrm{r}, 47.20 . \mathrm{Bp}, 47.52 .+\mathrm{j}, 47.27 . \mathrm{T}-$

\section{INTRODUCTION}

Spatiotemporal chaos in Rayleigh-Bénard convection was discovered by Ahlers and Behringer [1], Maurer and Libchaber [2], and Gollub and Benson [3]. In 1978, Ahlers and Behringer reported that as a control parameter was increased (here the temperature difference between the top and bottom plates), they found a transition to aperiodic time dependence in heat transport measurements of cylindrical RayleighBénard convection cells for a variety of aspect ratios. They also noted that as the aspect ratio $\Gamma$ [defined in Eq. (4)] was increased, the critical value of the control parameter for this transition decreased. Maurer and Libchaber studied rectangular convection cells and discovered frequency locking followed by a period doubling cascade to chaos, as the same control parameter was increased. They also observed hysteresis in these systems. Gollub and Benson performed visualizations on rectangular convection cells and found many of the signatures of chaos, including period-doubling bifurcations, entrainment, and intermittency. These three works provided strong evidence that Rayleigh-Bénard convection systems were chaotic for certain parameters. However, the Lyapunov exponents for experimentally realistic systems have never been computed. We will show that these systems are indeed chaotic in the sense of having a positive Lyapunov exponent.

We also test if the claims in the Egolf et al. [4] study can be generalized. Egolf et al. computed the Lyapunov spectral density for large aspect ratio Rayleigh-Bénard systems which exhibit spiral defect chaos (SDC). They demonstrated that the Lyapunov spectral density was extensive in that it scaled with system size. However, they used periodic boundary conditions. Lyapunov exponents have never been computed for realistic boundary conditions or for any other RayleighBénard convection system.

The Egolf paper also looked at visualizations of the perturbation field corresponding to the largest Lyapunov

\footnotetext{
*Electronic address: jscheel@caltech.edu
}

exponent (this perturbation field is also known as the Lyapunov eigenvector), and demonstrated that the regions where the perturbation grows are relatively localized and are correlated with dynamical events such as roll breaking or reconnection. They also found that these dynamical events were correlated with large spikes in the short-time dynamics of the leading order Lyapunov exponent. The Egolf paper makes the conclusion that the mechanism for generating chaotic dynamics in SDC is roll breaking or reconnection.

We will focus here on the experiments by Ahlers and Behringer [1,5-7] for small aspect ratio cylindrical cells $(\Gamma=2.08$ and 4.72$)$ in addition to the experiments performed by Gollub and Benson [3] for small rectangular cells (dimension $3.50 \times 2.08)$. We have published our results for larger aspect ratio $(10<\Gamma<40)$ elsewhere [8], since we used the larger aspect ratio simulations to study rotating RayleighBénard convection. However, the larger aspect ratio systems show incredibly complicated spatiotemporal dynamics with numerous defects and domain walls. We wanted to focus here on smaller aspect ratio cells, where the creation and annihilation of defects are more isolated, to see if the claim about the mechanism of chaos is true in general.

We show the results of our detailed investigation of the contribution of dislocation creation and annihilation events to the leading order Lyapunov exponent $\lambda_{1}$. While we do find that dislocation events are highly correlated with the short time dynamics of $\lambda_{1}$, we find that not all dislocation events are associated with contributions to the long time average of $\lambda_{1}$. We will also discuss the quantity $S_{\lambda}$, the running sum of the logarithm of the stretching, which we find is more useful for understanding the chaotic dynamics than either the shorttime Lyapunov exponent or the long-time average Lyapunov exponent. We find the short-time Lyapunov exponent can be somewhat misleading, since it can fluctuate wildly even when the overall average Lyapunov exponent is either zero or negative. Blindly computing the long-time average Lyapunov exponent can also be misleading, since transient dynamics can make a significant contribution to the exponent, or require much longer evolution times for convergence. 


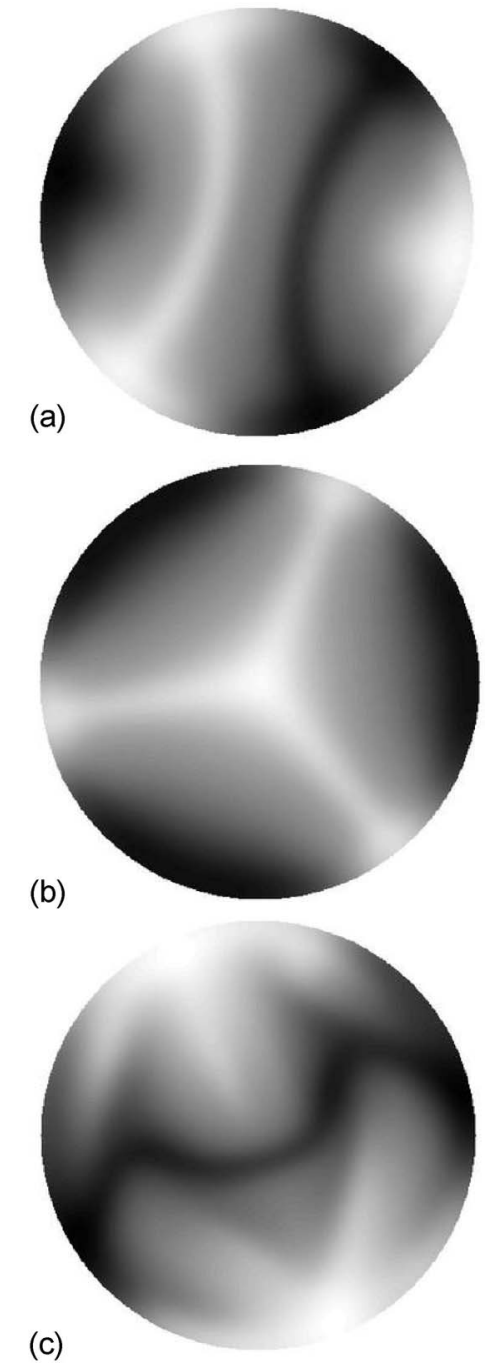

FIG. 1. Snapshot of temperature deviation $\theta$ at the midplane, for $\Gamma=2.08, \sigma=0.78$ and (a) $R=10000, t=20$, (b) $R=10000, t=66.2$, (c) $R=18800, t=29.3$. Lateral temperature boundary conditions are insulating. The gray denotes the conduction value $(\theta=0)$, and the lighter and darker shades give the values above and below this.

\section{NUMERICAL METHODS}

\section{A. Boussinesq equations}

The system is modeled by the Boussinesq equations [9], which consist of a modified Navier-Stokes equation (1), the heat equation (2), and incompressibility (3). The equations are

$$
\begin{gathered}
\sigma^{-1}\left(\partial_{t}+\mathbf{u} \cdot \nabla\right) \mathbf{u}=-\nabla P+\nabla^{2} \mathbf{u}+\theta \hat{z}, \\
\left(\partial_{t}+\mathbf{u} \cdot \nabla\right) \theta=\nabla^{2} \theta+R w \\
\nabla \cdot \mathbf{u}=0 .
\end{gathered}
$$

The variables are nondimensionalized by specifying the length in terms of the cell height $d$, the temperature in terms of temperature difference between the top and bottom plate $\Delta T$, and the time in units of the vertical thermal diffusion time $\tau_{v}=d^{2} / \kappa$, where $\kappa$ is the thermal diffusivity. The vari- able $\mathbf{u}(\mathbf{r}, t)$ is the velocity field, $P(\mathbf{r}, t)$ is the pressure, and $\theta(\mathbf{r}, t)$ is the temperature deviation from the linear conduction profile. The symbol $\partial_{t}$ indicates time differentiation and $\hat{z}$ is a unit vector in the vertical direction. The Prandtl number $\sigma=\nu / \kappa$, where $\nu$ is the kinematic viscosity. The Rayleigh number $R=\alpha g \Delta T d^{3} / \kappa \nu$, where $\alpha$ is the thermal expansion coefficient and $g$ is the acceleration of gravity.

The aspect ratio for cylindrical regions is defined as

$$
\Gamma=\frac{\text { radius }}{\text { depth }} .
$$

The control parameter $\epsilon$ is defined as

$$
\epsilon=\frac{R-R_{c}}{R_{c}},
$$

where $R_{c}$ is the critical Rayleigh number at which conduction gives way to convection.

We use no-slip velocity boundary conditions along the walls (as written in a cylindrical coordinate system)

$$
u_{r}=u_{\phi}=u_{z}=0 \quad \text { at } r=\Gamma, \quad \text { and } \quad z=0,1 .
$$

For our temperature boundary conditions, we use constant values along the top and bottom plates

$$
\theta=0, \quad \text { at } z=0,1 .
$$

For the sidewalls we use either conducting

$$
\theta=0 \quad \text { at } r=\Gamma
$$

or insulating thermal boundary conditions

$$
\partial_{r} \theta=0 \quad \text { at } r=\Gamma .
$$

To solve the Boussinesq equations, we have used the code Nek5000, a highly efficient, parallel, spectral element code developed to solve the Navier-Stokes equation. The details of the code are described elsewhere [10-13].

Ahlers and Behringer [1] measured heat transport using the Nusselt number, defined as the ratio of the total heat transported across the cell divided by the heat transported via conduction only. Hence if the Nusselt number is one, there is only conduction, and if the Nusselt number is greater than 1, there is convection as well. To allow for a simpler comparison, the reduced Nusselt number $N$ is studied: this is the Nusselt number minus one, which goes through zero at the onset of convection.

\section{B. Computation of Lyapunov exponents}

The leading order Lyapunov exponent is defined as the measure of the exponential stretching of the nearby trajectories $\mathbf{y}(t)$ and $\mathbf{y}(t)+\delta \mathbf{y}(t)$. We define $\mathbf{y}(t)=[\mathbf{u}, \theta]$ as the $(4 \times N)$-dimensional array describing the state of the $N$ fluid elements as a function of time and $\delta \mathbf{y}(t)=[\delta \mathbf{u}(t), \delta \theta(t)]$ as the corresponding perturbation field. The leading order Lyapunov exponent is then found by averaging over the entire fluid volume, as described below. The pressure $P$ can be determined from $\mathbf{y}$ and the perturbation pressure $\delta P$ can be determined from $\delta \mathbf{y}$. 


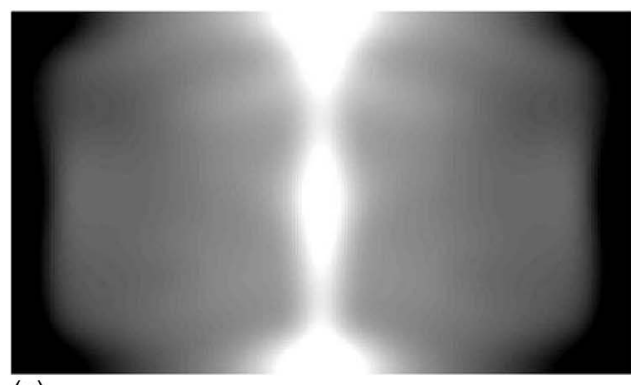

(a)

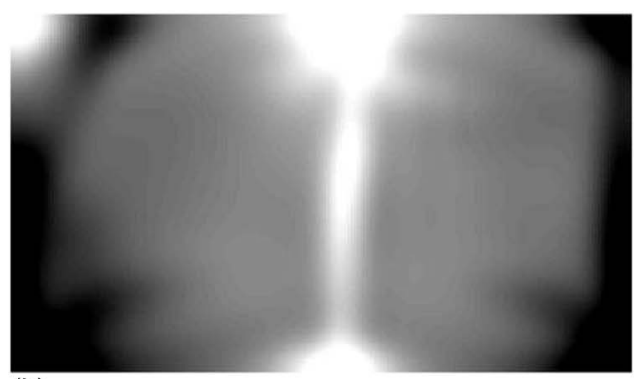

(b)

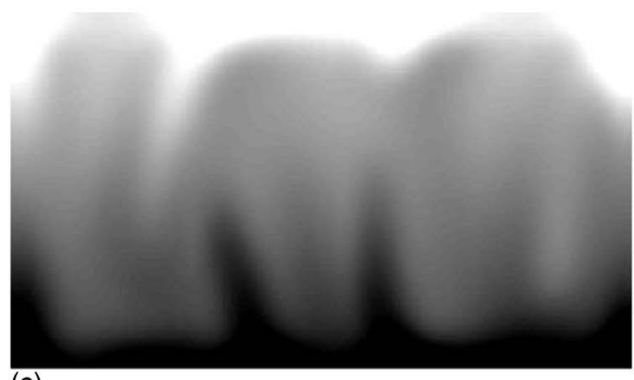

(c)

FIG. 2. Same as Fig. 1 but for a rectangular cell of dimensions $3.50 \times 2.08, \sigma=2.5$ and (a) $R=42500, t=25.78$, (b) $R=52110$, $t=54.2$, (c) $R=56000, t=24.1$. Lateral temperature boundary conditions are insulating. The power spectral density of these states is shown in Fig. 3.

If two states of the fluid differed initially by the small amount $\delta \mathbf{y}(0)$, then we can track the evolution of the perturbation by the linearized equations

$$
\frac{d \delta \mathbf{y}}{d t}=\mathbf{J}[\mathbf{y}(t)] \delta \mathbf{y},
$$

where $\mathbf{J}$ is the Jacobian of the system. If we define the full field evolution equations (1)-(3) as $d \mathbf{y}(t) / d t=\mathbf{F}(\mathbf{y})$ then $\mathbf{J}=\delta \mathbf{F} / \delta \mathbf{y}$. In our case, the specific equations are

$$
\begin{gathered}
\sigma^{-1}\left[\left(\partial_{t}+\mathbf{u} \cdot \nabla\right) \delta \mathbf{u}+(\delta \mathbf{u} \cdot \nabla) \mathbf{u}\right]=-\nabla \delta P+\nabla^{2} \delta \mathbf{u}+\delta \theta \hat{z}, \\
\left(\partial_{t}+\mathbf{u} \cdot \nabla\right) \delta \theta+(\delta \mathbf{u} \cdot \nabla) \theta=\nabla^{2} \delta \theta+R \delta w,
\end{gathered}
$$$$
\boldsymbol{\nabla} \cdot \delta \mathbf{u}=0 .
$$

The leading Lyapunov exponent $\lambda_{1}$ is then defined as [14-18]

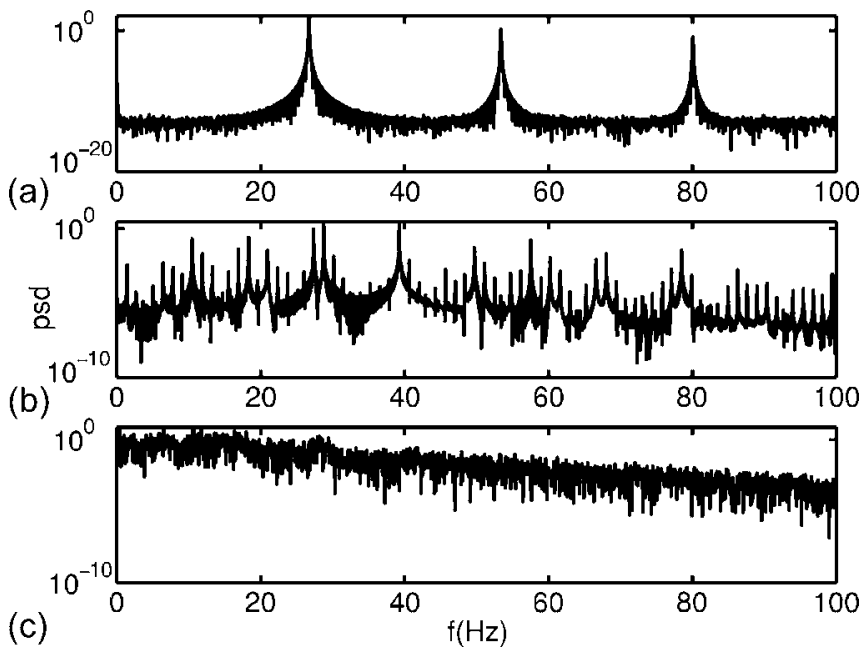

FIG. 3. The power spectral density of the Nusselt number as a function of frequency for the states shown in Fig. 2.

$$
\lambda_{1}=\lim _{t \rightarrow \infty} \frac{1}{t} \ln \left(\frac{\|\delta \mathbf{y}(t)\|}{\|\delta \mathbf{y}(0)\|}\right),
$$

where the normalization is defined over the interior volume $V$ as

$$
\|\delta \mathbf{y}(t)\|=\sqrt{\frac{1}{V} \int_{V}\left[R_{c} \delta \mathbf{u}(t)^{2}+\sigma \delta \theta(t)^{2}\right] d V .}
$$

We have chosen the above normalization to be consistent with the inner product used by Cross [19] in his analytical work.

Likewise, the instantaneous leading Lyapunov exponent is defined as $[4,8]$

$$
\lambda_{1}^{\text {inst }}=\frac{d}{d t} \ln \left(\frac{\|\delta \mathbf{y}(t)\|}{\|\delta \mathbf{y}(0)\|}\right) .
$$

The above quantity is useful for studying how $\lambda_{1}$ varies over short time intervals.

Another useful quantity is $S_{\lambda}$, which we define as

$$
S_{\lambda}=\ln \left(\frac{\|\delta \mathbf{y}(t)\|}{\|\delta \mathbf{y}(0)\|}\right) .
$$

The slope of $S_{\lambda}$ versus time gives an approximate value for $\lambda_{1}$ and the derivative of $S_{\lambda}$ versus time is $\lambda_{1}^{\text {inst }}$.

We find $\lambda_{1}$ and $\lambda_{1}^{\text {inst }}$ by numerically integrating the full fields (1)-(3) concurrently with the perturbation fields (11). We start the initial conditions for the full fields as small, random values, and for the perturbation field as random values as well (about 100 times smaller). We find it useful to renormalize the perturbation fields after a certain amount of time, to avoid overflow errors. Then we compute $S_{\lambda}$ from the normalization factors [20].

\section{RESULTS}

The results shown here use the same parameters as the experiments by Ahlers and Behringer [1] and Gollub and 


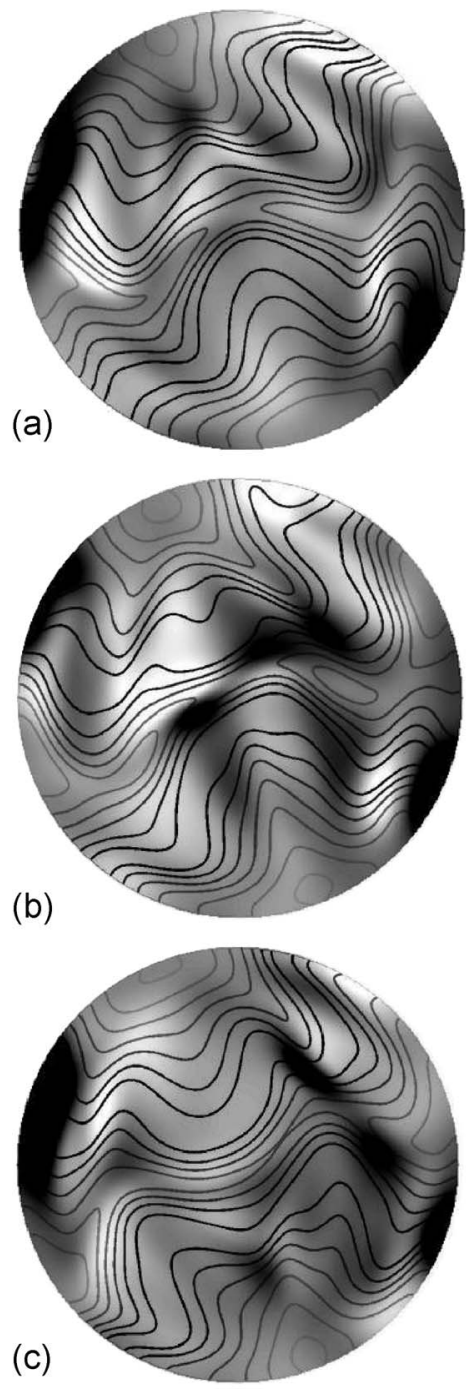

FIG. 4. Overlay of a grayscale density plot of the midplane temperature perturbation field $\delta \theta$ (same color scheme as in Fig. 1) and a contour plot of the midplane full temperature field $\theta$. The parameters are $\Gamma=2.08, \sigma=0.78, R=18800$, (a) $t=29.36$, (b) 29.39, (c) 29.43. Lateral temperature boundary conditions are insulating. A visualization of the temperature field is given in Fig. 1(c).

Benson [3]. For the experiments by Ahlers and Behringer, we use a Prandtl number $\sigma$ of 0.78 and investigate aspect ratios $\Gamma=2.08$ and 4.72. We use a time resolution $d t$ of 0.001 and a spatial resolution $d x$ of 0.1 for $\Gamma=4.72$, and a $d t$ of 0.0001 and $d x$ of 0.05 for $\Gamma=2.08$. For the experiments by Gollub and Benson we use a rectangular cell of dimensions $3.50 \times 2.08$, a $\sigma$ of 2.5 , insulating boundary conditions, a $d t$ of 0.0001 , and a $d x$ of 0.08 .

We verified convergence both with respect to spatial and temporal resolutions. We also verified that our results were independent of the initial perturbation field (see Ref. [11] for details). We chose our resolution to give us an accuracy of $1 \times 10^{-3}$ for the evolution of our fluid elements (this achieved the best balance between accuracy and integration time). Note that the error in the Lyapunov exponent will be somewhat larger since it requires an average over long evolution times and the convergence can be rather noisy. We needed a finer resolution for the smaller aspect ratios, since the Rayleigh numbers required for chaos were much larger, as shown in Fig. 9.

We will explore a variety of Rayleigh numbers $R$ both before and after the transition to aperiodic time dynamics. We use insulating thermal sidewall boundary conditions for the $\Gamma=2.08$ and rectangular cases, which most closely resembles the experiments $[7,11]$. We use conducting boundary conditions for the $\Gamma=4.72$ case to be consistent with Ref. [21], but the boundaries should matter less in the larger aspect ratio system.

\section{A. Visualizations}

Ahlers and Behringer did not perform any visualizations of their cells, so we have provided a few pictures of the smallest aspect ratio cells. A plethora of different states exists in these tiny cells as shown in Fig. 1. Note that Figs. 1(a) and 1 (b) correspond to the same $R$, but occur at different times. The three states have also been seen in recent experimental visualizations by Hof et al. [22], and computed by Borońska and Tuckerman [23] along with a variety of other planforms.

In Fig. 2 we show visualizations of the rectangular cells. The two-roll system shown in Figs. 2(a) and 2(b) are similar to those observed by Gollub and Benson. The noisy dynamics in Fig. 2(c) was not visualized by Gollub and Benson. It is interesting to note that the roll orientation has switched from Fig. 2(b) to 2(c). The power spectral density of the Nusselt number is reported in Fig. 3 for the corresponding Rayleigh numbers in Fig. 2. We find a periodic state for $R$ $=42500$ as evidenced by the single frequency in Fig. 3(a) (and its harmonics). We find a quasiperiodic state at $R$ $=52110$, since two incommensurate frequencies are present in Fig. 3(b). Finally we see a very broad power spectrum for the chaotic state at $R=56000$ as shown in Fig. 3(c). Similar power spectra were also observed by Gollub and Benson.

We have also found it productive to visualize the temperature perturbation field. When we looked at stationary systems we found the perturbation field to be extended, asymmetric and stationary. In contrast, we found that the perturbation field for systems with time dependence in the Nusselt number (either periodic or aperiodic) is characterized by localized bursts associated with some type of dynamical event such as defect creation or roll compression. This has also been seen by Egolf et al. [4] for larger aspect ratio (but using periodic boundary conditions), and for the parameter regime where SDC exists.

In Fig. 4 we have plotted an overlay of a grayscale density plot of the temperature perturbation field $\delta \theta$ and a contour plot of the full temperature field $\theta$. We plot an overlay to determine if localized activity in the perturbation field corresponds to certain features in the full field. For the parameters in Fig. 4, the system is undergoing oscillatory dynamics [24]. It oscillates between the state in Fig. 4(a) to its reflection in Fig. 4(c) and then back to a state which is similar to Fig. 4(a). The associated perturbation field shows a small, localized region of activity near the center whenever the system switches from one orientation to its inverted orientation. This can be seen in Fig. 4(b). This behavior is somewhat dwarfed 

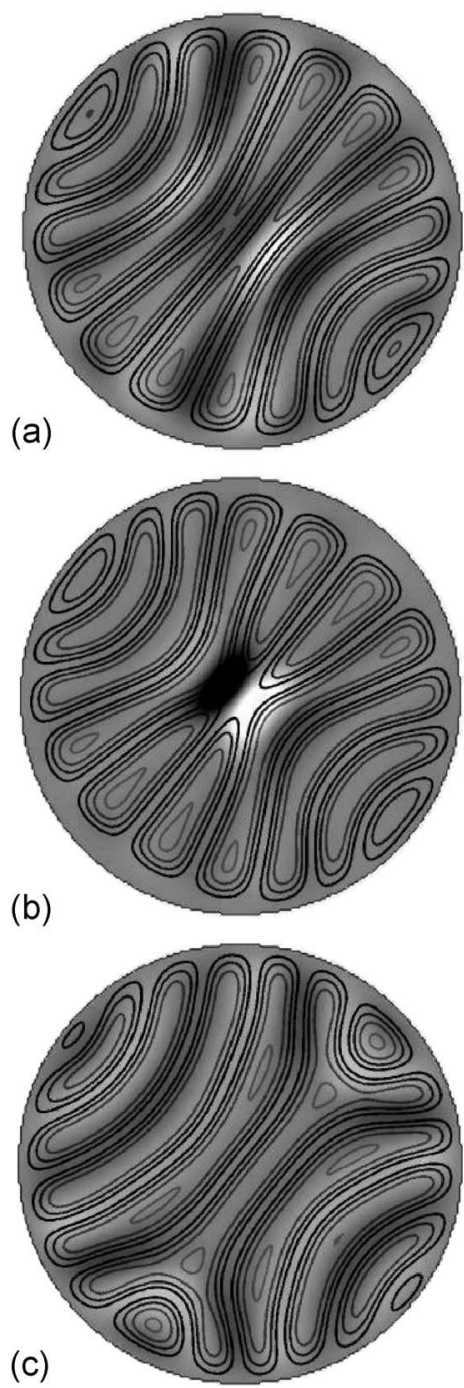

FIG. 5. Same type of plot as in Fig. 4 but for the following parameters: $\Gamma=4.72, \sigma=0.78, R=2800$, (a) $t=427.8$, (b) 438 , (c) 448.2. Lateral temperature boundary conditions are conducting.

by the extremely large activity near the edge of the cell. We do not have an explanation for the source of this activity.

In Fig. 5 we show a similar overlay plot for a $\Gamma=4.72$ cell. This time sequence shows that the creation of a dislocation pair is accompanied by a rather large spike in the perturbation field exactly where and when the defects are created.

Finally in Fig. 6 we show an overlay plot for the periodic state of the rectangular cell shown in Fig. 2(a). This periodic sequence starts with the two-roll state shown in Fig. 6(a). Then a "bubble" forms in the middle of the cell as shown in Fig. 6(b). Then this bubble climbs until it dissipates as shown in Fig. 6(c). Then the sequence repeats. Notice that there is a large region of activity in the perturbation field when the bubble is formed in Fig. 6(b). We also see activity along the sidewalls which is associated with the roll distortion that occurs there. In all cases (Figs. 4-6), the increase in the perturbation field activity is associated with a spike in $\lambda_{1}^{\text {inst }}$.

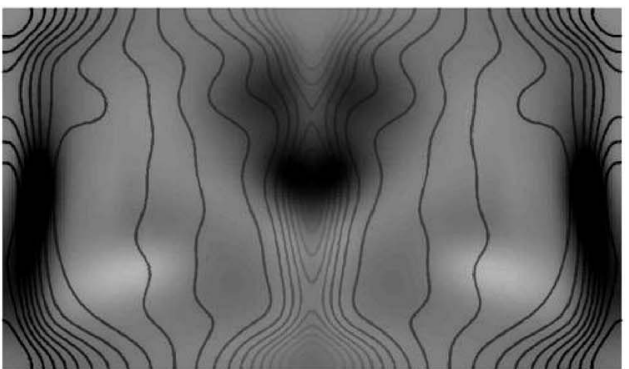

(a)

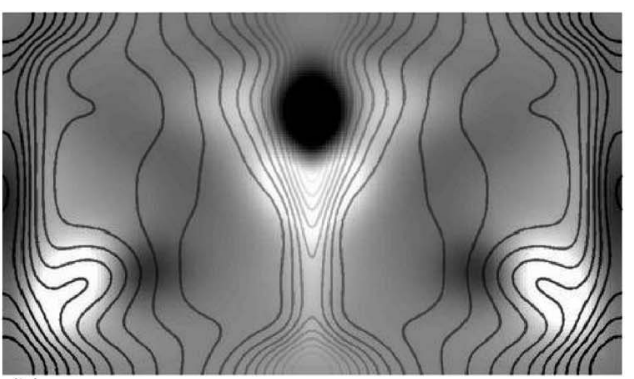

(b)

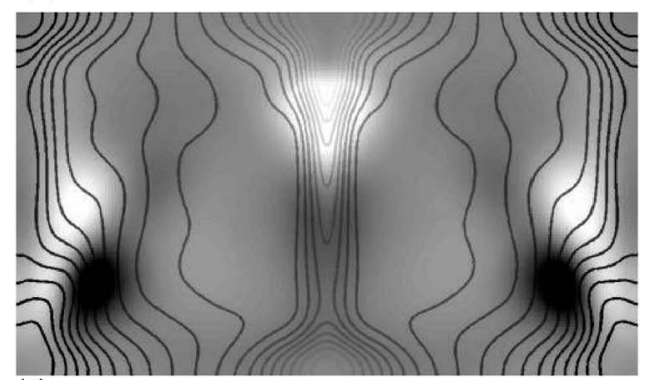

(c)

FIG. 6. Same type of plot as in Fig. 4 but for a rectangular cell of dimensions $3.50 \times 2.08, \sigma=2.5, R=42500$, and (a) $t=25.77$, (b) 25.78, (c) 25.79. Lateral temperature boundary conditions are insulating. A visualization of the temperature field is given in Fig. 2(a).

\section{B. Positive leading order Lyapunov exponents}

We computed the quantity $\lambda_{1}$ (12) for a variety of parameters, and found it to be positive in the régimes where Ahlers and Behringer and Gollub and Benson detected an aperiodic time dependence in $N$. For example, in Fig. 7 we have plotted $S_{\lambda}(15)$ versus time for $\Gamma=2.08, \sigma=0.78, R=18800$ and insulating boundary conditions (solid line). We see a general upward trend to $S_{\lambda}$ as a function of time, and the long time average slope is $\lambda_{1}=0.71 \pm 0.09$. This system shows chaotic behavior to at least 54 vertical diffusion times, which is quite long considering a horizontal diffusion time is equal to $\Gamma^{2} \approx 4$ for this case. The horizontal diffusion time is approximately the time it takes for a fluid particle to diffuse across the cell. We determined our error in $\lambda_{1}$ by dividing the time series $S_{\lambda}$ into approximately 6-8 equal parts, finding the slope, and hence $\lambda_{1}$ associated with each smaller time series. The mean of these values is close to the $\lambda_{1}$ found from the slope of the entire time series and the error is determined by finding the standard deviation of these values (divided by the square root of the number of values).

We also computed $\lambda_{1}$ for the larger aspect ratio case $\Gamma=4.72, \sigma=0.78, R=6950$ and with conducting boundary 


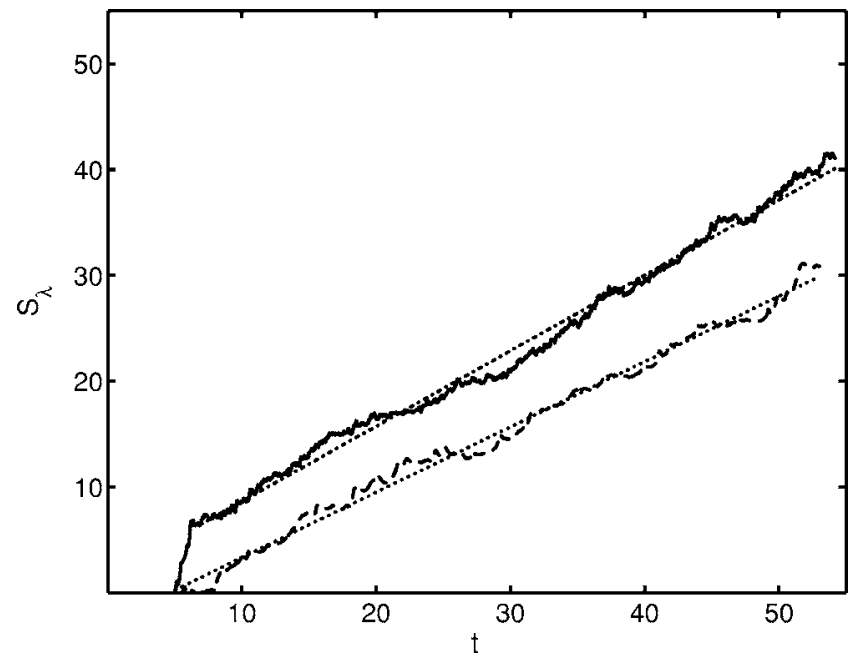

FIG. 7. The quantity $S_{\lambda}$ versus time. The slope of each graph converges to its respective $\lambda_{1}$, which is positive, indicating these systems are chaotic. We used the following parameters. Solid line: $\Gamma=2.08, \sigma=0.78, R=18800$, insulating boundaries. The associated dotted line is the slope taken from seven vertical diffusion times and up and gives $\lambda_{1}=0.71 \pm 0.09$ [a visualization of this state is shown in Figs. 1(c) and 4]. Dashed line: $\Gamma=4.72, \sigma=0.78, R=6950$, conducting boundaries. The slope (associated dotted line) is taken from 10 vertical diffusion times and up and gives $\lambda_{1}=0.62 \pm 0.06$.

conditions as shown in the dashed line of Fig. 7. Again we see a long time upward trend, and the slope is $\lambda_{1}=0.62 \pm 0.06$. Finally we computed $\lambda_{1}$ for the chaotic state of the rectangular cell of dimensions $3.50 \times 2.08, \sigma=2.5$, $R=56000$, and insulating boundary conditions. We find a very steady, very large long time upwards trend as shown in Fig. 8. The slope gives a $\lambda_{1}=7.3 \pm 0.3$, indicating the chaos is much stronger in this system. These positive exponents prove that these aperiodic systems are truly chaotic in the sense of exponential divergence of nearby trajectories.

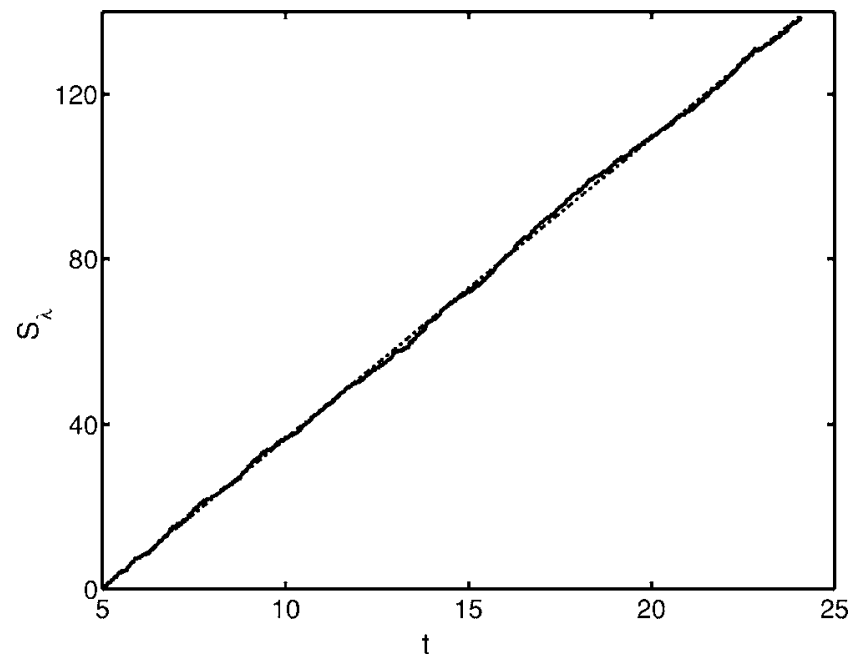

FIG. 8. The same as Fig. 7 but for a rectangular cell of dimensions $3.50 \times 2.08, \sigma=2.5, R=56000$, insulating boundaries. The slope (associated dotted line) is taken from seven vertical diffusion times and up and gives $\lambda_{1}=7.3 \pm 0.3$. A visualization of this state is shown in Fig. 2(c). (a)
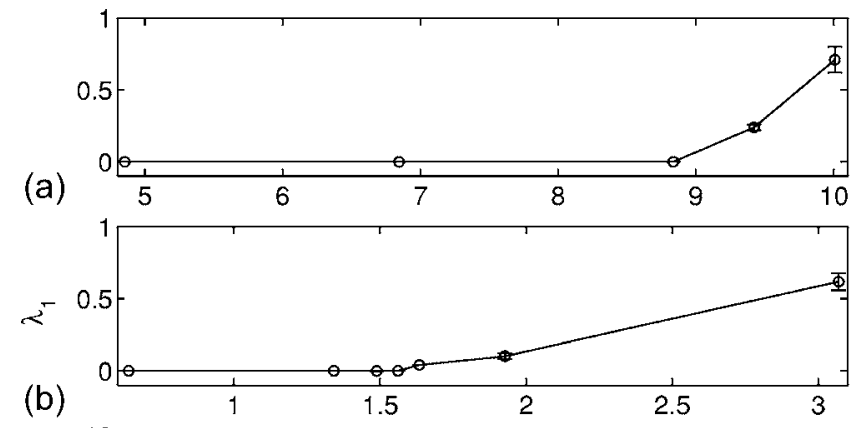

(b)

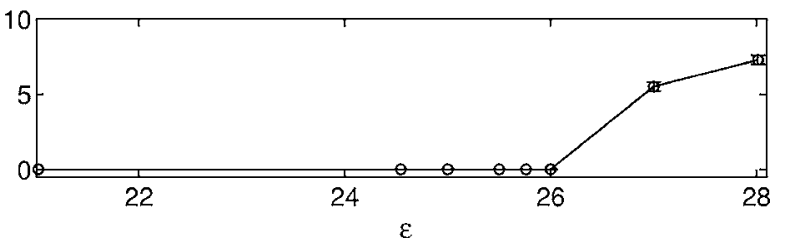

FIG. 9. The quantity $\lambda_{1}$ versus $\epsilon$ for the following: (a) $\Gamma=2.08$, $\sigma=0.78, R_{c}=1708$, insulating boundaries, (b) $\Gamma=4.72, \sigma=0.78$, $R_{c}=1708$, conducting boundaries, (c) rectangle of dimensions $3.50 \times 2.08, \sigma=2.5, R_{c}=1930$, insulating boundaries. In all cases the computed $\lambda_{1}$ is the value corresponding to the state the system converges to after a long period of time. Initial transient states and the contribution they make to $\lambda_{1}$ were neglected.

We have plotted the $\lambda_{1}$ values for variety of $\epsilon$ values in Figs. 9 and 10. We see similar behavior for all three of our convection cells. We find that $\lambda_{1}$ is zero when the cells exhibit periodic or quasiperiodic behavior (or at least zero within our limit of accuracy). We find $\lambda_{1}$ is positive, and increases with $\epsilon$ when the cells exhibit nonperiodic behavior. Note that the first data point in Fig. 9(a) does correspond to the steady state shown in Fig. 15 for $t>50$. However, all of the other data points correspond to either periodic or aperiodic dynamics. For the $\Gamma=2.08$ cell [Fig. 9(a)], we find periodic behavior for $4.8<\epsilon \leq 8.8$, and a transition to chaos somewhere between $8.8<\epsilon<9.4$. Likewise for $\Gamma=4.72$ [Fig. $9(\mathrm{~b})$ ], we find periodic behavior for $0.6<\epsilon \leq 1.56$ and a transition to chaotic behavior for $1.56<\epsilon<1.64$. These results are consistent with the results of Ahlers and Behringer who found a transition to chaos for $\epsilon \simeq 10$ for the $\Gamma=2.08$ cell and $\epsilon \simeq 1$ for the $\Gamma=4.72$ cell. Finally for our rectangular system [Fig. 9(c)] we find periodic behavior for $21<\epsilon \leq 26$ and a transition to chaos for $26<\epsilon<27$. Likewise these results are consistent with Gollub and Benson who found a transition to chaos for $\epsilon=27$.

Although we have computed a $\lambda_{1}$ value equal to zero for all periodic states, we find negative $\lambda_{1}$ values associated with steady states near threshhold, as is plotted in Fig. 10 for our rectangular cells. We also see a peak in the $\lambda_{1}$ as we go through thershhold, as is expected for a bifurcation [18].

We have also computed how $\lambda_{1}$ scales with $\epsilon$ and $\Gamma$ for our larger aspect ratios and rotating Rayleigh-Bénard convection [8]. We find that $\lambda_{1}$ scales linearly with $\epsilon$ as predicted theoretically [25]. We found an unexpected nonintensive scaling of $\lambda_{1}$ with $\Gamma$. We have not explored the scaling of $\lambda_{1}$ with $\epsilon$ or $\Gamma$ in any detail for our smaller aspect ratios, since the dynamics is much more variable in the smaller aspect ratios. For example, Hof [22] has shown that eight different states coexist for $\Gamma \simeq 2$. It would be very difficult to 


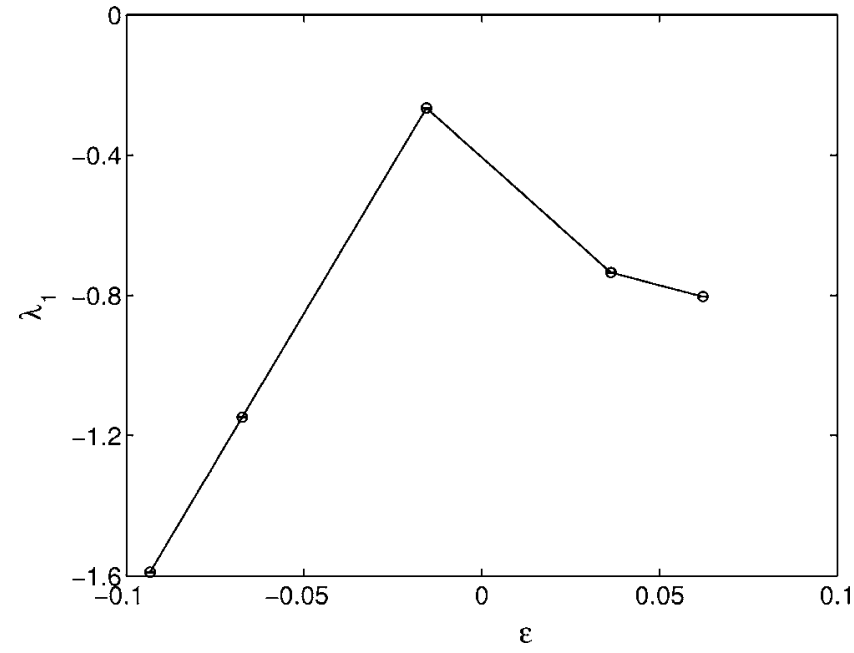

FIG. 10. The same as Fig. 9(c), but very near threshhold.

distinguish between trends as a result of a transition to a different state versus trends due to an increase in $R$ and $\Gamma$ for the same state. In addition, these systems often undergo transitions to different states after a long evolution time (see Fig. 15), making such a comparison doubly difficult.

\section{Short time dynamics of leading Lyapunov exponents}

We would like to use the short time dynamics of $\lambda_{1}$ to identify sources of chaos. It was suggested by Egolf et al. [4], who studied the SDC state of Rayleigh-Bénard convection, that roll breaking and reconnection events caused spikes in the short time Lyapunov exponent $\lambda_{1}^{\text {inst}}$, which in turn provided the most significant contributions to the long time Lyapunov exponent $\lambda_{1}$. By looking at small aspect ratio cells, we have determined that this claim is not true in general.

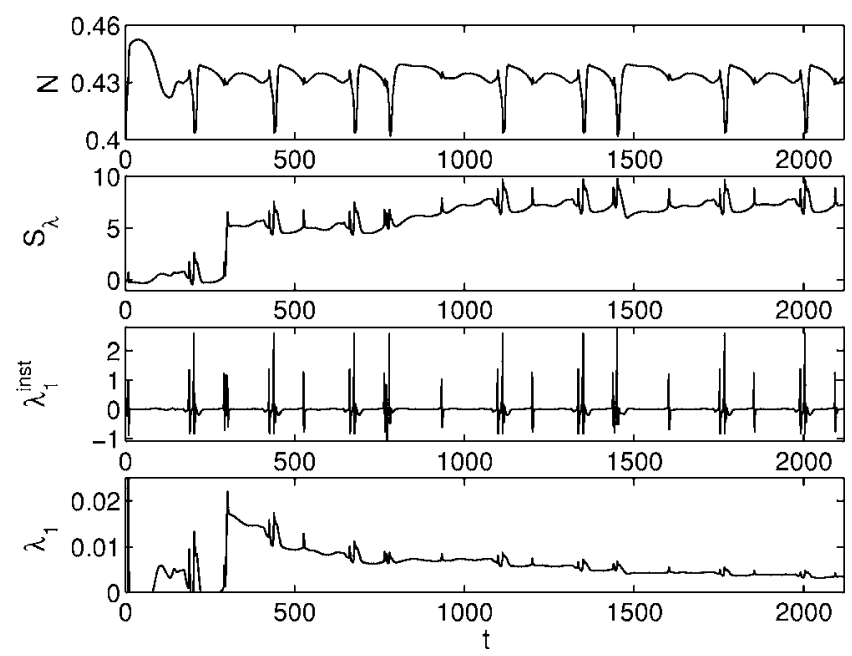

FIG. 11. First panel: Reduced Nusselt number $N$ as a function of time $t$ for $\Gamma=4.72, \sigma=0.78, R=2800$, conducting boundaries. Second panel: Corresponding $S_{\lambda}$ as a function of time $t$. Third panel: Corresponding $\lambda_{1}^{\text {inst }}$ as a function of time $t$. Fourth panel: Corresponding $\lambda_{1}$ as a function of time $t$. A visualization of this state is shown in Fig. 5.
We will analyze in detail a rather simple, almost periodic state for $\Gamma=4.72, \sigma=0.78$, and $R=2800$ (corresponding to $\epsilon=0.64)$. These states have already been investigated by Paul et al. [21] and the dynamics is shown in Fig. 11 for a long time series. The first panel shows the reduced Nusselt number $N$ as a function of time. One sees the pattern is almost periodic after $t=150$ vertical diffusion times. There is a characteristic pattern to the dislocation creation and annihilation events. For example, the large dip in the first panel at $t=438.36$ corresponds to the dislocation pair creation shown in Fig. 5(b), and is immediately followed by dislocation climb to the opposite sides of the container shown in Fig. 5(c). Then the dislocations slowly glide along the rim until they are annihilated successively. The annihilation of the first dislocation occurs at $t=526$, and the second dislocation is annihilated at $t=662$, just before the next creation event at $t=675.76$. Note that each dislocation event is associated with a spike in $S_{\lambda}$ (second panel). However, after the sequence occurs, there is little net rise in $S_{\lambda}$ [26]. This can be seen in the relatively flat regions of $S_{\lambda}$ in the second panel, such as $400<t<700$ and $1100<t<1400$. This indicates that while a dislocation creation/annihilation event is associated with a localized stretching of phase space, it is not associated with the long time Lyapunov exponent.

We have also plotted $\lambda_{1}^{\text {inst }}$ as a function of time in the third panel of Fig. 11. While each dislocation event does cause a burst of activity in $\lambda_{1}^{\text {inst }}$, it is very difficult to tell from looking at $\lambda_{1}^{\text {inst }}$ which dislocation events contribute to the longtime exponent $\lambda_{1}$, hence we prefer to look at $S_{\lambda}$ versus time. This is reinforced in the fourth panel where we show $\lambda_{1}$ versus time. The quantity $\lambda_{1}(t)$ is found by evaluating Eq.

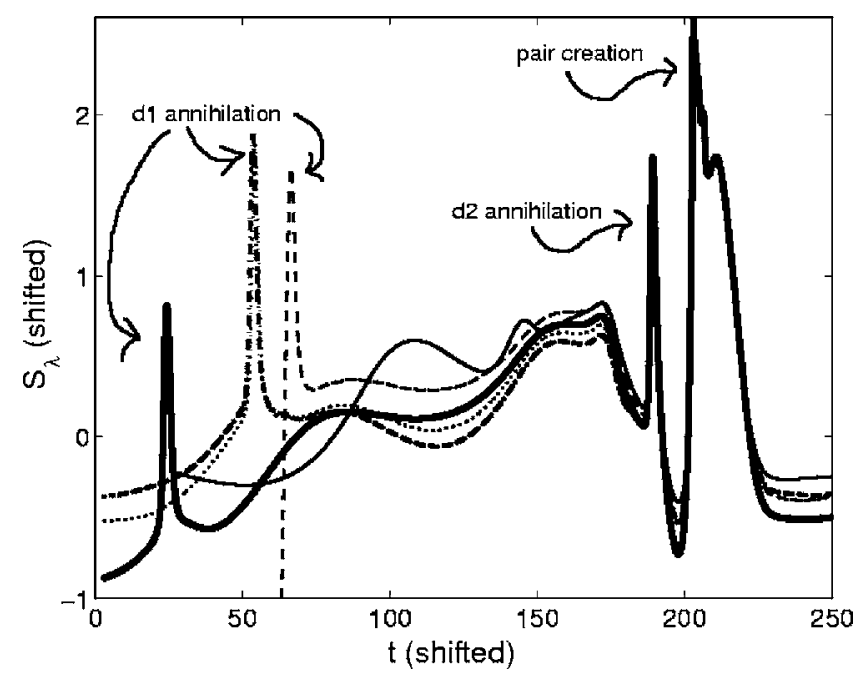

FIG. 12. Shifted $S_{\lambda}$ versus shifted $t$ to compare the events. Approximately 250 vertical diffusion time sections of the $S_{\lambda}$ versus $t$ plot in Fig. 11 have been shifted horizontally and vertically to coincide with the peak at $t=202.92$, corresponding to a dislocation pair creation. The defect annihilation peaks are also highlighted as " $d 1$," corresponding to the preceding first defect annihilation and " $d 2$," corresponding to the second. Legend: thin solid line, peak $t=202.92$, dashed line, peak $t=438.36$, dashed-dotted line, peak $t=675.76$, thick solid line, peak $t=1120$, dotted line, peak $t=1349.2$. 

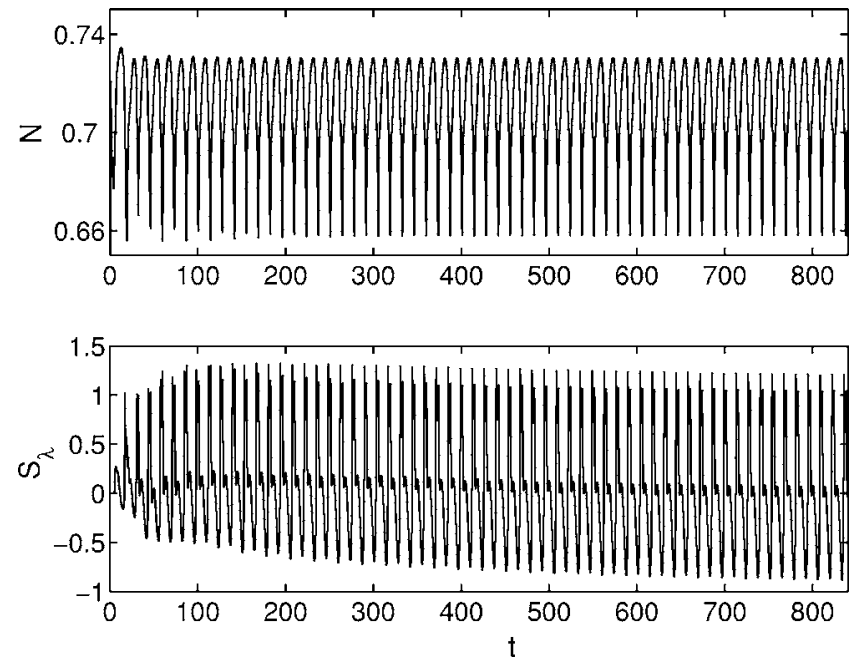

FIG. 13. Top panel: Reduced Nusselt number $N$ as a function of time $t$ for $\Gamma=4.72, \sigma=0.78, R=4000$, conducting boundaries. Bottom panel: Corresponding $S_{\lambda}$ as a function of time $t$.

(12) from some initial time $t_{\text {init }}$ to the time $t$. Note that the value of $\lambda_{1}$ remains positive, because of the large initial transients, including the one at $t=300$. It is much more accurate to find $\lambda_{1}$ from evaluating the slope of $S_{\lambda}$, selecting the region where the transients have died out. When we do this, we obtain $\lambda_{1}=0.000 \pm 0.001$ for the state shown in Fig. 11 .

We focus in more detail on the dislocation/creation events in Fig. 12, where successive time intervals of about 250 vertical diffusion times have been shifted horizontally and vertically to correspond to the dislocation pair creation peak at $t=202.92$. One sees that the dislocation pair creation events neatly overlap. One also sees a return to almost the exact same $S_{\lambda}$ value after the event. Hence one can conclude that these repeated dislocation events are not associated with a positive $\lambda_{1}$. If we compare the most regular cases, $t=675.76$ (dashed-dotted line) and $t=1329.2$ (dotted line), we see that even the preceding first dislocation annihilation events located near $t($ shifted $)=50$ overlap. However, note that the other preceding first dislocation annihilation events do not overlap (look at the thick solid line, corresponding to $t=1120$ and the dashed line corresponding to $t=438.36)$. Visualization of these regions show a different pattern to the events at those times. It is precisely these regions where one sees the largest rise in $S_{\lambda}$. The rise at $t=300$ does correspond to an unusual roll pinch-off event, however the rise between $900<t<1100$ only corresponds to a slower than average first dislocation annihilation. Likewise, at $t=800$, the first dislocation annihilation event is almost immediately followed by the second dislocation annihilation and pair creation events.

One sees further support for this by looking at other Rayleigh numbers. The results for $R=4000$ are plotted in Fig. 13. Here the defect creation and annihilation events arrive at a much higher frequency. We also see that after $t=100$ there is an almost perfect periodicity to the pattern, hence one finds no rise in $S_{\lambda}$, and possibly a very slight decline. Conversely, for systems exhibiting non-repeated events in the Nusselt number, one sees $S_{\lambda}$ exhibiting an overall positive slope (such as in Figs. 7 and 8).
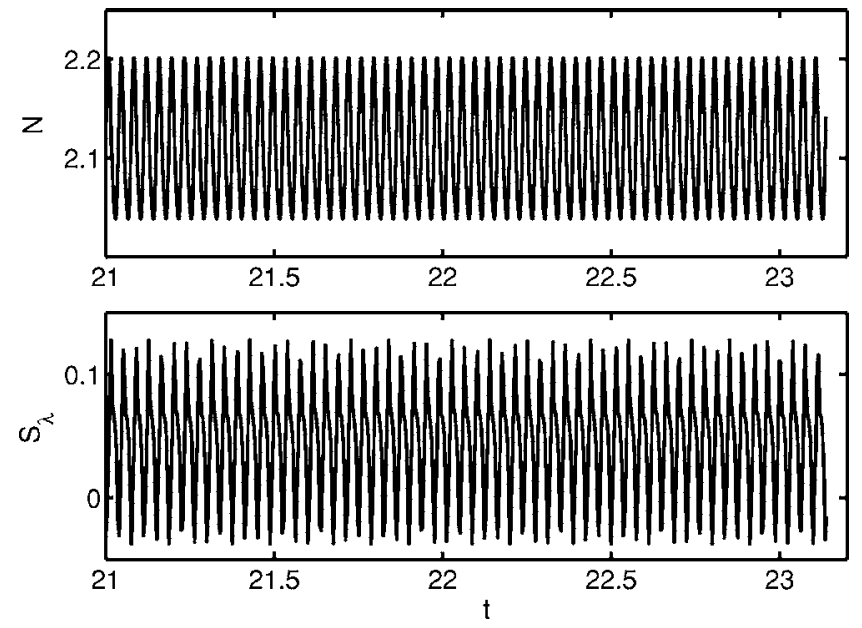

FIG. 14. Top panel: Reduced nusselt number $N$ as a function of time $t$ for a rectangular cell of dimensions $3.50 \times 2.08, \sigma=2.5, R$ $=42500$, insulating boundaries. Bottom panel: Corresponding $S_{\lambda}$ as a function of time $t$. Visualizations of this state are given in Figs. 2(a) and 6.

Finally we show the results for the periodic state of the rectangular cell in Fig. 14. This state does not involve a defect creation or annihilation event, but instead the roll compression event described in Fig. 6. We have only shown the last two vertical diffusion times, since the oscillation frequency is so large in this system. However, the system was run out from zero to 23 vertical diffusion times. We again see that a periodic system gives us a zero $\lambda_{1}$, even though the short time dynamics of $\lambda_{1}$ are very strong.

Hence we conclude that dynamical events such as dislocation creation/annihilation and roll compression events are not always associated with an overall net rise in $S_{\lambda}$ and hence $\lambda_{1}$. For all the cases we have studied (over twenty different cases, each ran out to at least fifteen vertical diffusion times, see Ref. [11] for details) we find that if the dynamical events
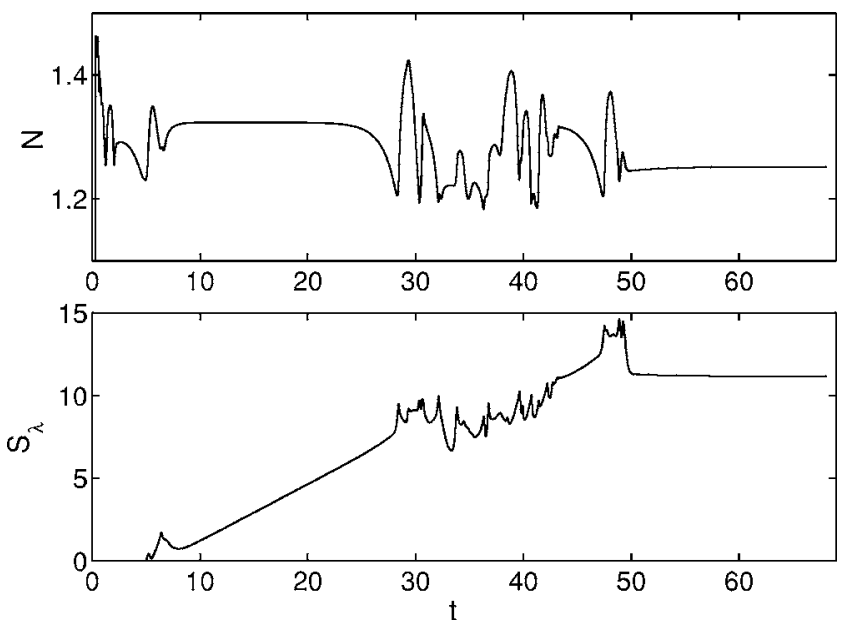

FIG. 15. Top panel: Reduced Nusselt number as a function of time $t$ for $\Gamma=2.08, \sigma=0.78, R=10000$, insulating boundaries. Bottom panel: Corresponding $S_{\lambda}$ versus time. A visualization of the temperature field is given in Figs. 1(a) and 1(b). 
are repeated, they are not associated with a positive $\lambda_{1}$, and if they are not repeated they are associated with a positive $\lambda_{1}$.

\section{Lyapunov exponents and stationary states}

We wanted to verify that our Lyapunov solver was working by studying $\lambda_{1}$ in the régime where a time-independent state was observed by Ahlers and Behringer. We choose an $R$ value of 10000 , well within the region of stationary, convecting states specified by Ahlers and Behringer. We first evolved the system out to about 20 vertical diffusion times and did observe $N$ converging to a constant value, as shown in the top panel of Fig. 15 for $t<20$. The system settles down to the roll state shown in Fig. 1(a). However, we were surprised to find that the quantity $S_{\lambda}$ had a positive slope as shown in the bottom panel of Fig. 15 for $t<20$. This indicated that the system was in a transient state. When we evolved the system even longer, we found that this parallel roll state evolved into a state with noisy dynamics, which lasted for another 20 vertical diffusion times. Then the system settled into another stationary state, this time the Mercedes-like pattern shown in Fig. 1(b). This state has a $\lambda_{1}$ that is very slightly negative. Since $\lambda_{1}$ is no longer positive, we can be assured that the system is now in its stationary state. Without the knowledge of $\lambda_{1}$, however, we would not be assured that this state is transient without running the system out for a much longer time.

\section{CONCLUSIONS}

We have shown that systems with an aperiodic time dependence, as observed by Ahlers and Behringer and Gollub and Benson, have positive leading order Lyapunov exponents, hence they are indeed chaotic as defined by the exponential divergence of nearby trajectories.

We have also visualized the evolution of the perturbation field. For cases associated with a time-dependent Nusselt number, the perturbation field consists of localized regions of disturbances which coincide with some dynamical event. Conversely perturbation fields corresponding to stationary systems are extended, asymmetric and stationary.

Finally we have compared the correlations between $S_{\lambda}$ and hence $\lambda_{1}$ with dynamical events such as defect creation/ annihilation and roll compression events. While repeated dy- namical events are associated with a temporary change in the stretching rate of phase space, we do not find there to be an overall net increase in $S_{\lambda}$ and hence a positive $\lambda_{1}$. We find instead that nonrepeated events are associated with a positive $\lambda_{1}$. Hence the suggestion by Egolf that roll breaking and reconnection events contribute significantly to the largest Lyapunov exponent is incorrect, at least in a general sense. Merely looking at $\lambda_{1}^{\text {inst }}$ is insufficient to determine chaotic dynamics. It is more productive to instead look at the long time evolution of $S_{\lambda}$.

It is possible that one could have chaotic/nonperiodic rotations of a pattern in a circular cell that would not show up in the volume averaged leading order Lyapunov exponents computed in this paper. It would be very interesting to extend the numerical techniques developed in this paper to compute the other positive Lyapunov exponents.

In the future we would like to investigate the contributions to $S_{\lambda}$ in more detail. We wish to determine what precisely causes $S_{\lambda}$ to rise, such as a slow drift due to mean flow or the timing between events or something else entirely.

\section{ACKNOWLEDGMENTS}

The authors thank Paul Fischer and Anand Jayaraman for the use of their numerical code NEK5000, which was used for all simulations. They would like to thank Mark Paul, KengHwee Chiam, and Henry Greenside for helpful discussions. This work was supported by the Engineering Research Program of the Office of Basic Energy Sciences at the Department of Energy, Grant Nos. DE-FG03-98ER14891 and DEFG02-98ER14892. The numerical code was run on the following supercomputing sites, whom the authors gratefully acknowledge: the National Energy Research Scientific Computing Center which is supported by the Office of Science of the U.S. Department of Energy under Contract No. DE-AC03-76SF00098, the Center for Computational Sciences at Oak Ridge National Laboratory, which is supported by the Office of Science of the Department of Energy under Contract No. DE-AC05-00OR22725, "Jazz," a 350-node computing cluster operated by the Mathematics and Computer Science Division at Argonne National Laboratory as part of its Laboratory Computing Resource Center, and the IBM "Blue Gene" system at the San Diego Supercomputing Center.
[1] G. Ahlers and R. P. Behringer, Phys. Rev. Lett. 40, 712 (1978).

[2] J. Maurer and A. Libchaber, J. Phys. (France) Lett. 40, L419 (1979).

[3] J. P. Gollub and S. V. Benson, J. Fluid Mech. 100, 449 (1980).

[4] D. A. Egolf, I. M. Melnikov, W. Pesch, and R. E. Ecke, Nature (London) 404, 733 (2000).

[5] G. Ahlers, Phys. Rev. Lett. 33, 1185 (1974).

[6] B. P. Behringer and G. Ahlers, Phys. Lett. 62A, 329 (1977).

[7] R. P. Behringer and G. Ahlers, J. Fluid Mech. 125, 219 (1982).

[8] A. Jayaraman, J. D. Scheel, H. S. Greenside, and P. F. Fischer, Phys. Rev. E 74, 016209 (2006).
[9] M. C. Cross and P. C. Hohenberg, Rev. Mod. Phys. 65, 851 (1993).

[10] P. F. Fischer, J. Comput. Phys. 133, 84 (1997).

[11] J. D. Scheel, Ph.D. thesis, California Institute of Technology, Pasadena, 2006.

[12] J. D. Scheel, M. R. Paul, M. C. Cross, and P. F. Fischer, Phys. Rev. E 68, 066216 (2003).

[13] J. D. Scheel and M. C. Cross, Phys. Rev. E 72, 056315 (2005).

[14] A. Wolf, J. B. Swift, H. L. Swinney, and J. A. Vastano, Physica D 16, 285 (1985).

[15] K. Geist, U. Parlitz, and W. Lauterborn, Prog. Theor. Phys. 83, 
875 (1990).

[16] J. P. Eckmann and D. Ruelle, Rev. Mod. Phys. 57, 617 (1985).

[17] J. P. Eckmann, S. O. Kamphorst, D. Ruelle, and S. Ciliberto, Phys. Rev. A 34, 4971 (1986).

[18] L. N. Hand and J. D. Finch, Analytical Mechanics (Cambridge University Press, Cambridge, 1998).

[19] M. C. Cross, Phys. Fluids 23, 1727 (1980).

[20] T. S. Parker and L. O. Chua, Practical Numerical Algorithms for Chaotic Numerical Systems (Springer-Verlag, Berlin, 1989).
[21] M. R. Paul, M. C. Cross, P. F. Fischer, and H. S. Greenside, Phys. Rev. Lett. 87, 154501 (2001).

[22] B. Hof, G. J. Lucas, and T. Mullin, Phys. Fluids 11, 2815 (1999).

[23] K. Borońska and L. S. Tuckerman (unpublished).

[24] R. M. Clever and F. H. Busse, J. Fluid Mech. 176, 403 (1987).

[25] Y. Tu and M. C. Cross, Phys. Rev. Lett. 69, 2515 (1992).

[26] We have found that the behavior of $S_{\lambda}$ is relatively insensitive to the choice of initial conditions $\delta \mathbf{y}(0)$. See Ref. [11] for more details. 